\title{
GAIA accuracy on radial velocities assessed from a synthetic spectra database
}

\author{
T. Zwitter \\ University of Ljubljana, Department of Physics, Jadranska 19, 1000 Ljubljana, Slovenia \\ Received 29 October 2001 / Accepted 14 February 2002

\begin{abstract}
Spectrograph aboard the GAIA satellite operates in the near-IR, in the 8490-8740 $\AA$ window accessible also from the ground. The most important parameter yet to be determined is the spectral resolution. Realistic estimates of the zodiacal light background are obtained and a total of $2 \times 10^{5}$ correlation runs are used to study the accuracy of radial velocity measured by the spectrograph as a function of resolution, magnitude of the target, its spectral type and luminosity class. Accuracy better than $2 \mathrm{~km} \mathrm{~s}^{-1}$ is achievable for bright stars if a high enough dispersion is chosen. Radial velocity error of $5 \mathrm{~km} \mathrm{~s}^{-1}$ is at $V=17.5$ for Cepheids and at 17.7 for horizontal branch stars. Even for very faint objects, with spectra dominated by background and readout noise, the optimal dispersion is still in the $0.25 / 0.75 \AA /$ pix range. This is also true for complicated cases such as spectroscopic binaries or if information other than radial velocity, i.e. abundances of individual elements or stellar rotation velocity, is sought after. The results can be scaled to assess performance of future ground based instruments.
\end{abstract}

Key words. stars: kinematics - surveys - space vehicles: instruments - techniques: radial velocities

\section{Introduction}

GAIA is the approved ESA Cornerstone 6 mission designed to obtain extremely precise astrometry (in the micro-arcsec regime), multi-band photometry as well as spectroscopy for up to a billion stars in our Galaxy and beyond. The goals are described in the mission Concept and technology study report (ESA-SCI (2000) 4, hereafter ESA 2000), and summarized by Perryman et al. (2001). Astrometric information will be used to derive star's position and distance. Proper motion then yields the projection of the velocity vector on the sky plane. The missing, sixth component in the position-velocity space, i.e. the radial velocity of the target, is derived by spectroscopic observations on board (Munari 1999). Apart from astrometry and spectroscopy a number of photometric bands (15 are currently baselined) will collect broad- and narrow-band photometric information useful for general classification purposes (see Sect. 3.3). Spectroscopy can be used to obtain additional astrophysical information that cannot be provided by other means. This includes abundances of individual elements, stellar rotational velocity $(v \sin i)$, and information on line profiles which is essential for derivation of orbital solutions of spectroscopic binaries (Munari et al. 2001a) or for detection and classification of peculiar stars (Munari et al. 2002). The spectrograph is described in ESA 2000 and Munari (2001).

\footnotetext{
* e-mail: tomaz.zwitter@uni-lj.si
}

Performance of the spectrograph can be assessed by convolution of spectra of real stars (Munari et al. 2001). Here we resort to synthetic spectra that give more flexibility to simulate parameters of the GAIA spectrograph. The goal of this paper is to assess the accuracy of the radial velocities and to some extent other information as a function of the chosen spectral dispersion. We upgrade the initial assessment (ESA 2000) by a database of more realistic spectra, a better treatment of the background, better correlation technique, updated spectrograph parameters and extending results to significantly fainter magnitudes. Most important, the initial assessment was done for only one resolution $(R=30000$ sampled at $0.75 \AA /$ pixel, i.e. an undersampled case) while here we explore a range of possible choices $(2000<R<20000$, always maintaining the usual criterion of 2 pixels per resolution element). Given the fact that the spectral resolution is the only spectrograph parameter yet to be decided and the possibility to scale the results to any ground-based telescope observing in the near-IR, the paper provides realistic error estimates for any future campaigns in the near-IR domain.

\section{Synthetic spectra database}

We used Kurucz models to calculate a large database of $\sim 10^{5}$ synthetic spectra that sample the grid in temperature, surface gravity, metallicity, rotational velocity and spectral resolution. The grid which will be described 


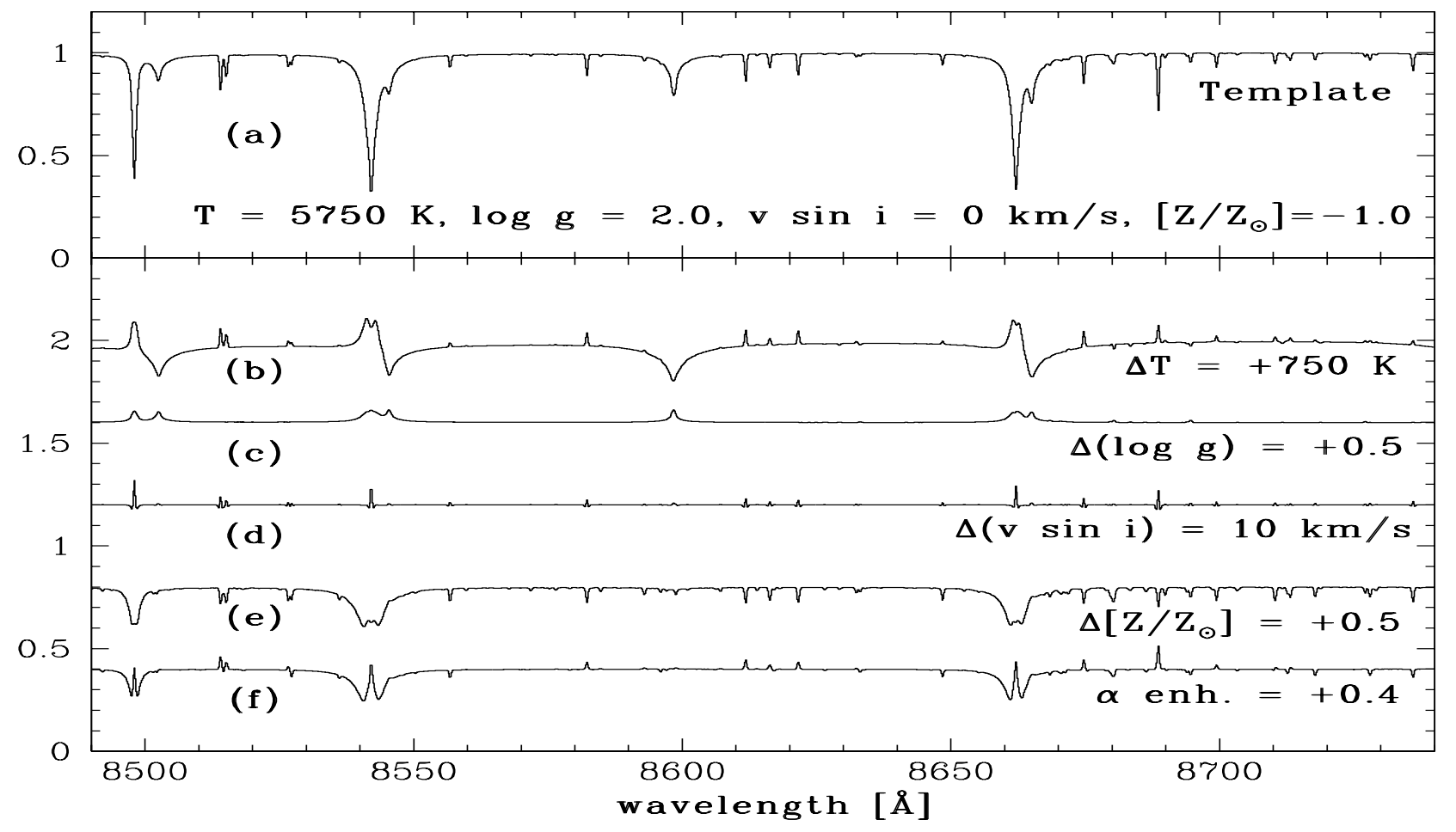

Fig. 1. a) Synthetic spectrum of a non-rotating G-type metal-deficient giant at a $R=20000$ resolution. b)-f) Spectral change if one of the spectral parameters is modified. Each curve is the ratio of the given spectrum divided by the top template. The values of the changing parameter are indicated. All tracings share the scale of the top panel, but are vertically offset for clarity.

separately (Zwitter et al. 2002) upgrades the initial calculations of Munari \& Castelli (2000) and Castelli \& Munari (2001). Figure 1 illustrates the dependence of spectral properties on basic model parameters. All tracings are ratios to the template spectrum of a non-rotating metal-deficient giant of spectral type $\mathrm{G}$, which is shown in Fig. 1a. Plotting the ratio to the chosen template instead of the spectrum itself permits a quick evaluation of the signal to noise and resolution needed to determine the value of a given parameter. Rise of effective temperature weakens the Ca II lines while the Pashen lines get stronger (Fig. 1b). Increase of gravity (Fig. 1c) also diminishes the width of the Ca II lines. Stellar rotation (Fig. 1d) has a pronounced effect only for fast rotators $\left(v \sin i>10 \mathrm{~km} \mathrm{~s}^{-1}\right)$, but the smearing is different from that of increase in gravity. The metallicity content (Fig. 1e) influences many lines, but details are different from that of enrichment with the $\alpha$-elements (Fig. 1f). These kind of dependencies are typical for relatively low temperature stars that will be the most common GAIA targets.

In the next section we use few of the calculated spectra to assess the performance of the spectrograph for some representative targets.

\section{Simulations}

\subsection{Spectrograph characteristics}

Radial velocity spectrometer instrument aboard GAIA is a slitless spectrograph operating in a time delay integration mode. The field of view is $2^{\circ} \times 1^{\circ}$. The telescope has a rectangular aperture of $0.75 \mathrm{~m} \times 0.7 \mathrm{~m}$. The focal plane is covered with an array of CCDs. The total efficiency of the telescope + spectrograph + CCD is baselined to 0.35. Each star drifts across the plane in $60.4 \mathrm{~s}$, with its signal read by two consecutive CCDs. The CCDs have $20 \mu \mathrm{m}$ pixels, corresponding to 1 arcsec on the sky. The readout noise of each CCD is $3.0 \mathrm{e}^{-}$. The dispersion direction is perpendicular to the drift. Each star has all signal on one or two pixels in the drift direction. It was suggested to study a possibility of binning together the pixels in the drift direction for faint objects and so avoid excessive RON. The background is calculated from a suitable region displaced along the direction of the drift scan. Altogether the number of pixels sent to the Earth is $3 \times 0.06 R$ for bright stars, and $2 \times 0.06 R$ for faint stars binned across the spatial direction (if the binning is used).

\subsection{Noise sources}

For bright targets the results will be limited by Poisson noise, but at the faint limit the read-out noise and noise of the background become more important. GAIA will operate from the second Lagrangian point of the Sun-Earth system. Save for the densest sky regions the dominant background signal will be zodiacal light (ESA 2000, see also Sect. 5 below). Here we explore its spatial variation in some detail.

The zodiacal background observed by GAIA will be much the same as seen from Earth, as the cloud of 


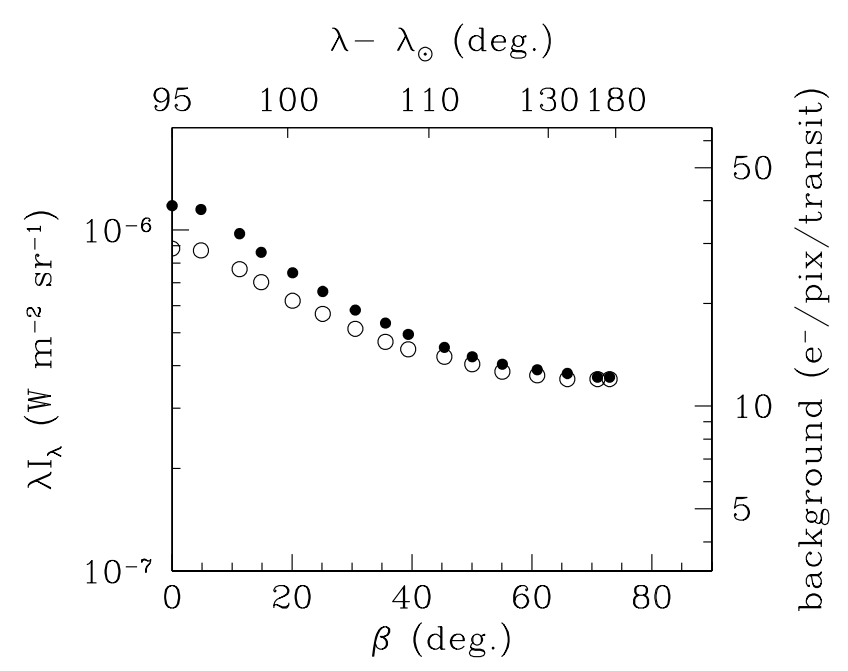

Fig. 2. Ecliptic latitude dependence of the zodiacal background for the $0.5 \mu \mathrm{m}$ (filled symbols) and $1.43 \mu \mathrm{m}$ (open symbols) bands. Adapted from Matsumoto et al. (1996). The scale on the right gives the corresponding number of electrons per pixel per observation detected by GAIA.

scattering particles extends well beyond the GAIA orbit (Kelsall et al. 1998; Gorkavyi et al. 2000). Observations of the Infrared Telescope in Space (Matsumoto et al. 1996) show that sky brightness at 0.5 and at $1.43 \mu \mathrm{m}$ has very similar energy density and similar dependence on the viewing direction (Fig. 2). This is consistent with earlier balloon observations at $0.71 \mu \mathrm{m}$ and $0.82 \mu \mathrm{m}$ (Frey et al. 1974). The angular distribution of the zodiacal brightness is however remarkably un-uniform. It depends on ecliptic coordinates of the viewing direction (here we neglect minor asymmetries vs. ecliptic plane, see James et al. 1997). Figure 3a shows the number of zodiacal light photons $\left(N_{\text {sky }}\right)$ per pixel detected during a single $60.4 \mathrm{~s}$ transit. The number is calculated from data in Levasseur-Regourd \& Dumont (1980) using the parameters of the spectrograph cited above and assuming that a suitable filter blocks away any light outside the GAIA spectral window. The numbers show that the zodiacal background noise $\left(\sqrt{N_{\text {sky }}}\right)$ is larger than the readout noise for two readouts $\left(3.0 \sqrt{2} \mathrm{e}^{-}\right)$, except near the ecliptic poles where the two contributions are comparable.

GAIA spectroscope always points perpendicularly to the axis of the satellite, which is aimed at the angle of $55^{\circ}$ to the Sun. So the sky is not sampled uniformly. Figure $3 \mathrm{~b}$ shows that directions at the angle of $90^{\circ} \pm 55^{\circ}$ to the Sun are sampled more often than the ones in between. As the telescope circles the Sun each star will have its helioecliptic longitude drifting across the whole range, but its ecliptic latitude remains constant.

Note that the readout noise and the zodiacal background (per pixel) do not depend on the chosen spectral resolution, while the stellar signal per pixel is inversely proportional to the spectral resolution. So for faint targets the background and read-out noise degrade the results more if the spectral resolution is increased.

\subsection{Single transit and mission average spectra}

We ran $2.4 \times 10^{5}$ simulations to evaluate performance of the GAIA spectrograph in measuring stellar radial velocities. Three cases were considered: a relatively hot A8 type main sequence star, a cooler K1/2 main sequence star and a G6 type supergiant. These choices are representative of the most important GAIA targets: a hot relatively luminous star that can be seen up to galactocentric distance, a cool dwarf of most common type observed by GAIA and a luminous supergiant that can be seen even in other galaxies.

A spectrum of the object was degraded to a desired spectral resolution and assigned the flux corresponding to its $V$ magnitude. The spectrum was then Doppler shifted to a randomly chosen velocity in the $\pm 100 \mathrm{~km} \mathrm{~s}^{-1}$ range. After addition of the zodiacal background the Poisson noise and the readout noise (corresponding to two consecutive CCD readouts during a single transit) were added. The radial velocity was recovered by correlation with a synthetic spectrum (Tonry \& Davis 1979). Correlations were done with the xcsao task within the IRAF's rvsao package (Kurtz et al. 1992). Degradation of spectral resolution, rebinning, Doppler shifts etc. were performed with a custom IRAF script.

It would be unphysical to try to recover the radial velocity using the same template as was used for initial input. GAIA photometric observations will provide quite an accurate guess on the stellar parameters of the target, but the match will never be perfect. Typical errors after the end of the mission will be $125 \mathrm{~K}$ in temperature, 0.4 in $\left[Z / Z_{\odot}\right]$ and 0.25 in $\log g$ (ESA 2000). To stay on the conservative side and to allow for a limited ability to reproduce observed spectra by theoretical calculation we used a larger mismatch: the spectrum used to recover the radial velocity had a random mismatch of $250 \mathrm{~K}$ in temperature, 0.5 in $\left[Z / Z_{\odot}\right]$ and 0.5 in $\log g$. The rotational velocity was also different: the initial input spectrum had $v \sin i=20 \mathrm{~km} \mathrm{~s}^{-1}$ (for $\mathrm{A} 8 \mathrm{~V}$ and $\mathrm{K} 1 / 2 \mathrm{~V}$ star), and $2 \mathrm{kms}^{-1}$ (G6 I star), while the one used as a correlation template had $v \sin i=10 \mathrm{~km} \mathrm{~s}^{-1}$ (for A $8 \mathrm{~V}$ and $\mathrm{K} 1 / 2$ $\mathrm{V}$ star), and $5 \mathrm{~km} \mathrm{~s}^{-1}$ (G6 I star).

Apart from a spectrum obtained during a single passage over the target we also simulated mission-averaged spectra. On the average each target will be observed 100 times during the 4-year mission. So we co-added 100 single mission spectra in order to simulate the ability of deriving radial velocities for single non-varying stars. The conditions for such summation will be met: each spectrum (and not only the correlation function) will be sent to the Earth, and previewed accuracy of wavelength calibration will permit their co-addition at the end of the mission.

\section{Results}

Results of the achievable radial velocity accuracy are presented in Fig. 4 and Tables 1 and 2. Each point 
Table 1. Radial velocity error (in $\mathrm{km} \mathrm{s}^{-1}$ ) for a background of $74 \mathrm{e}^{-}$per wavelength bin, typical for an object in the ecliptic plane. Values are given for mission averaged spectra (100 transits) while those in brackets are for a single transit spectrum.

\begin{tabular}{|c|c|c|c|c|c|c|c|c|}
\hline \multirow[t]{2}{*}{ Sp. Type } & \multirow{2}{*}{$\begin{array}{l}\text { Dispersio } \\
\AA / \text { pix } \\
\end{array}$} & $R$ & \multicolumn{6}{|c|}{ Visual magnitude } \\
\hline & & $\lambda / \Delta \lambda$ & 14.0 & 15.0 & 16.0 & 17.0 & 18.0 & 19.0 \\
\hline \multirow[t]{5}{*}{$\mathrm{A} 8 \mathrm{~V}$} & 0.25 & 17200 & $1.58(5.43)$ & $1.90(24.0)$ & $3.06(>40)$ & $7.84(>40)$ & $>40(>40)$ & $>40(>40)$ \\
\hline & 0.50 & 8610 & $2.80(6.31)$ & $3.01(17.0)$ & $3.79(>40)$ & $6.62(>40)$ & $32.2(>40)$ & $>40(>40)$ \\
\hline & 0.75 & 5740 & $4.66(7.92)$ & $4.69(17.7)$ & $5.15(>40)$ & $7.72(>40)$ & $26.0(>40)$ & $>40(>40)$ \\
\hline & 1.00 & 4305 & $6.81(10.9)$ & $6.87(16.6)$ & $7.15(>40)$ & $9.69(>40)$ & $22.1(>40)$ & $>40(>40)$ \\
\hline & 2.00 & 2160 & $13.2(16.5)$ & $13.4(24.5)$ & $13.9(>40)$ & $15.9(>40)$ & $25.6(>40)$ & $>40(>40)$ \\
\hline \multirow[t]{5}{*}{$\mathrm{K} 1 \mathrm{~V}$} & 0.25 & 17200 & $0.23(1.84)$ & $0.40(4.17)$ & $0.92(14.9)$ & $2.30(>40)$ & $5.59(>40)$ & $>40(>40)$ \\
\hline & 0.50 & 8610 & $0.28(2.10)$ & $0.44(4.38)$ & $0.96(9.97)$ & $2.10(>40)$ & $5.24(>40)$ & $19.4(>40)$ \\
\hline & 0.75 & 5740 & $0.29(2.53)$ & $0.47(5.06)$ & $1.08(10.5)$ & $2.23(>40)$ & $5.45(>40)$ & $13.8(>40)$ \\
\hline & 1.00 & 4305 & $0.31(3.00)$ & $0.56(5.50)$ & $1.11(12.5)$ & $2.54(39.7)$ & $5.72(>40)$ & $15.3(>40)$ \\
\hline & 2.00 & 2160 & $0.57(5.20)$ & $0.93(9.52)$ & $1.67(17.3)$ & $3.35(39.1)$ & $7.18(>40)$ & $19.1(>40)$ \\
\hline \multirow[t]{5}{*}{ G6 I } & 0.25 & 17200 & $0.11(1.09)$ & $0.20(2.41)$ & $0.44(5.92)$ & $1.09(>40)$ & $2.86(>40)$ & $25.9(>40)$ \\
\hline & 0.50 & 8610 & $0.17(1.52)$ & $0.31(3.37)$ & $0.65(7.41)$ & $1.52(32.7)$ & $3.48(>40)$ & $12.4(>40)$ \\
\hline & 0.75 & 5740 & $0.48(1.88)$ & $0.55(3.60)$ & $0.82(7.71)$ & $1.72(27.2)$ & $4.11(>40)$ & $11.9(>40)$ \\
\hline & 1.00 & 4305 & $0.67(2.28)$ & $0.77(4.25)$ & $1.07(8.31)$ & $1.98(23.7)$ & $4.54(>40)$ & $11.4(>40)$ \\
\hline & 2.00 & 2160 & $0.63(3.55)$ & $0.77(6.45)$ & $1.25(11.4)$ & $2.74(28.2)$ & $5.69(>40)$ & $13.5(>40)$ \\
\hline \multirow[t]{5}{*}{ Cepheid } & 0.25 & 17200 & $0.34(2.60)$ & $0.59(5.09)$ & $1.28(22.3)$ & $3.14(>40)$ & $7.48(>40)$ & $>40(>40)$ \\
\hline & 0.50 & 8610 & $0.38(2.86)$ & $0.60(5.34)$ & $1.15(13.4)$ & $2.70(>40)$ & $6.52(>40)$ & $24.6(>40)$ \\
\hline & 0.75 & 5740 & $0.63(3.34)$ & $0.78(5.64)$ & $1.32(12.2)$ & $2.85(>40)$ & $6.56(>40)$ & $18.6(>40)$ \\
\hline & 1.00 & 4305 & $0.71(3.32)$ & $0.87(6.77)$ & $1.34(13.2)$ & $2.98(31.9)$ & $6.58(>40)$ & $18.1(>40)$ \\
\hline & 2.00 & 2160 & $0.63(5.24)$ & $0.97(9.09)$ & $1.86(17.0)$ & $3.54(35.0)$ & $7.50(>40)$ & $19.8(>40)$ \\
\hline \multirow[t]{5}{*}{ HB } & 0.25 & 17200 & $0.34(2.19)$ & $0.52(4.48)$ & $1.07(20.8)$ & $2.51(>40)$ & $7.30(>40)$ & $>40(>40)$ \\
\hline & 0.50 & 8610 & $0.57(2.51)$ & $0.72(5.32)$ & $1.21(16.4)$ & $2.64(>40)$ & $6.00(>40)$ & $33.8(>40)$ \\
\hline & 0.75 & 5740 & $0.90(3.09)$ & $1.00(5.95)$ & $1.47(13.2)$ & $2.75(>40)$ & $6.44(>40)$ & $21.6(>40)$ \\
\hline & 1.00 & 4305 & $1.35(3.98)$ & $1.45(6.24)$ & $1.77(14.0)$ & $2.95(>40)$ & $6.66(>40)$ & $23.8(>40)$ \\
\hline & 2.00 & 2160 & $2.32(7.28)$ & $2.52(10.2)$ & $2.93(20.9)$ & $4.63(>40)$ & $9.47(>40)$ & $22.8(>40)$ \\
\hline
\end{tabular}

Table 2. Radial velocity error (in $\mathrm{km} \mathrm{s}^{-1}$ ) for a background of $20 \mathrm{e}^{-}$per wavelength bin, typical for an object at ecliptic latitude $\sim 45^{\circ}$. Values are given for mission averaged spectra (100 transits) while those in brackets are for a single transit spectrum.

\begin{tabular}{|c|c|c|c|c|c|c|c|c|}
\hline \multirow[t]{2}{*}{ Sp. Type } & \multirow{2}{*}{$\begin{array}{l}\text { Dispersion } \\
\AA / \text { pix }\end{array}$} & R & \multicolumn{6}{|c|}{ Visual magnitude } \\
\hline & & $\lambda / \Delta \lambda$ & 14.0 & 15.0 & 16.0 & 17.0 & 18.0 & 19.0 \\
\hline \multirow[t]{5}{*}{$\mathrm{A} 8 \mathrm{~V}$} & 0.25 & 17200 & $1.64(4.63)$ & $1.67(15.4)$ & $2.13(>40)$ & $3.98(>40)$ & $14.2(>40)$ & $>40(>40)$ \\
\hline & 0.50 & 8610 & $2.82(5.60)$ & $2.82(11.0)$ & $3.21(>40)$ & $4.16(>40)$ & $8.98(>40)$ & $>40(>40)$ \\
\hline & 0.75 & 5740 & $4.64(7.54)$ & $4.65(11.8)$ & $4.96(35.8)$ & $5.73(>40)$ & $9.57(>40)$ & $>40(>40)$ \\
\hline & 1.00 & 4305 & $6.78(10.1)$ & $6.76(13.9)$ & $6.92(32.7)$ & $7.73(>40)$ & $12.4(>40)$ & $31.2(>40)$ \\
\hline & 2.00 & 2160 & $13.2(18.0)$ & $13.4(22.9)$ & $13.6(39.9)$ & $14.4(>40)$ & $18.0(>40)$ & $36.0(>40)$ \\
\hline \multirow[t]{5}{*}{ K1 V } & 0.25 & 17200 & $0.19(1.52)$ & $0.28(3.01)$ & $0.53(6.82)$ & $1.19(39.4)$ & $2.86(>40)$ & $8.26(>40)$ \\
\hline & 0.50 & 8610 & $0.25(1.83)$ & $0.36(3.32)$ & $0.61(7.10)$ & $1.24(21.9)$ & $2.92(>40)$ & $7.44(>40)$ \\
\hline & 0.75 & 5740 & $0.26(2.32)$ & $0.42(4.11)$ & $0.74(7.58)$ & $1.32(20.2)$ & $3.15(>40)$ & $7.37(>40)$ \\
\hline & 1.00 & 4305 & $0.27(2.98)$ & $0.45(5.23)$ & $0.86(8.81)$ & $1.66(28.6)$ & $3.38(>40)$ & $7.93(>40)$ \\
\hline & 2.00 & 2160 & $0.60(4.85)$ & $0.80(8.52)$ & $1.38(13.5)$ & $2.42(27.2)$ & $5.22(>40)$ & $10.4(>40)$ \\
\hline \multirow[t]{5}{*}{ G6 I } & 0.25 & 17200 & $0.10(0.98)$ & $0.14(1.86)$ & $0.28(4.05)$ & $0.59(26.4)$ & $1.50(>40)$ & $3.96(>40)$ \\
\hline & 0.50 & 8610 & $0.15(1.38)$ & $0.24(2.36)$ & $0.50(4.78)$ & $0.95(14.3)$ & $2.06(>40)$ & $4.54(>40)$ \\
\hline & 0.75 & 5740 & $0.47(1.70)$ & $0.52(3.02)$ & $0.67(5.79)$ & $1.07(14.9)$ & $2.51(>40)$ & $5.58(>40)$ \\
\hline & 1.00 & 4305 & $0.69(2.11)$ & $0.71(3.60)$ & $0.85(6.77)$ & $1.36(14.7)$ & $2.77(>40)$ & $6.08(>40)$ \\
\hline & 2.00 & 2160 & $0.59(3.79)$ & $0.75(5.72)$ & $1.05(10.1)$ & $1.76(19.2)$ & $3.34(>40)$ & $7.85(>40)$ \\
\hline \multirow[t]{5}{*}{ Cepheid } & 0.25 & 17200 & $0.31(2.16)$ & $0.48(4.09)$ & $0.90(9.37)$ & $2.05(>40)$ & $4.87(>40)$ & $16.6(>40)$ \\
\hline & 0.50 & 8610 & $0.36(2.47)$ & $0.48(4.59)$ & $0.89(8.79)$ & $1.80(28.0)$ & $4.18(>40)$ & $11.1(>40)$ \\
\hline & 0.75 & 5740 & $0.59(2.72)$ & $0.72(4.80)$ & $1.04(10.1)$ & $1.94(21.2)$ & $4.40(>40)$ & $11.5(>40)$ \\
\hline & 1.00 & 4305 & $0.69(3.55)$ & $0.81(5.81)$ & $1.17(9.89)$ & $2.00(22.9)$ & $4.41(>40)$ & $11.1(>40)$ \\
\hline & 2.00 & 2160 & $0.58(5.49)$ & $0.95(8.32)$ & 1.59 (13.9) & $2.85(28.5)$ & $5.28(>40)$ & $11.6(>40)$ \\
\hline \multirow[t]{5}{*}{$\mathrm{HB}$} & 0.25 & 17200 & $0.31(1.75)$ & $0.44(3.70)$ & $0.79(8.97)$ & $1.68(>40)$ & $4.06(>40)$ & $23.0(>40)$ \\
\hline & 0.50 & 8610 & $0.52(2.31)$ & $0.64(4.22)$ & $0.92(8.69)$ & $1.81(37.5)$ & $4.27(>40)$ & $12.1(>40)$ \\
\hline & 0.75 & 5740 & $0.88(3.13)$ & $1.03(4.79)$ & $1.22(9.76)$ & $2.02(36.0)$ & $4.67(>40)$ & $11.1(>40)$ \\
\hline & 1.00 & 4305 & $1.38(3.78)$ & $1.41(6.16)$ & $1.65(10.2)$ & $2.45(28.2)$ & $4.81(>40)$ & $11.6(>40)$ \\
\hline & 2.00 & 2160 & $2.37(6.89)$ & $2.46(10.1)$ & $2.92(17.8)$ & $4.04(37.7)$ & $7.08(>40)$ & $15.9(>40)$ \\
\hline
\end{tabular}




\section{a)}

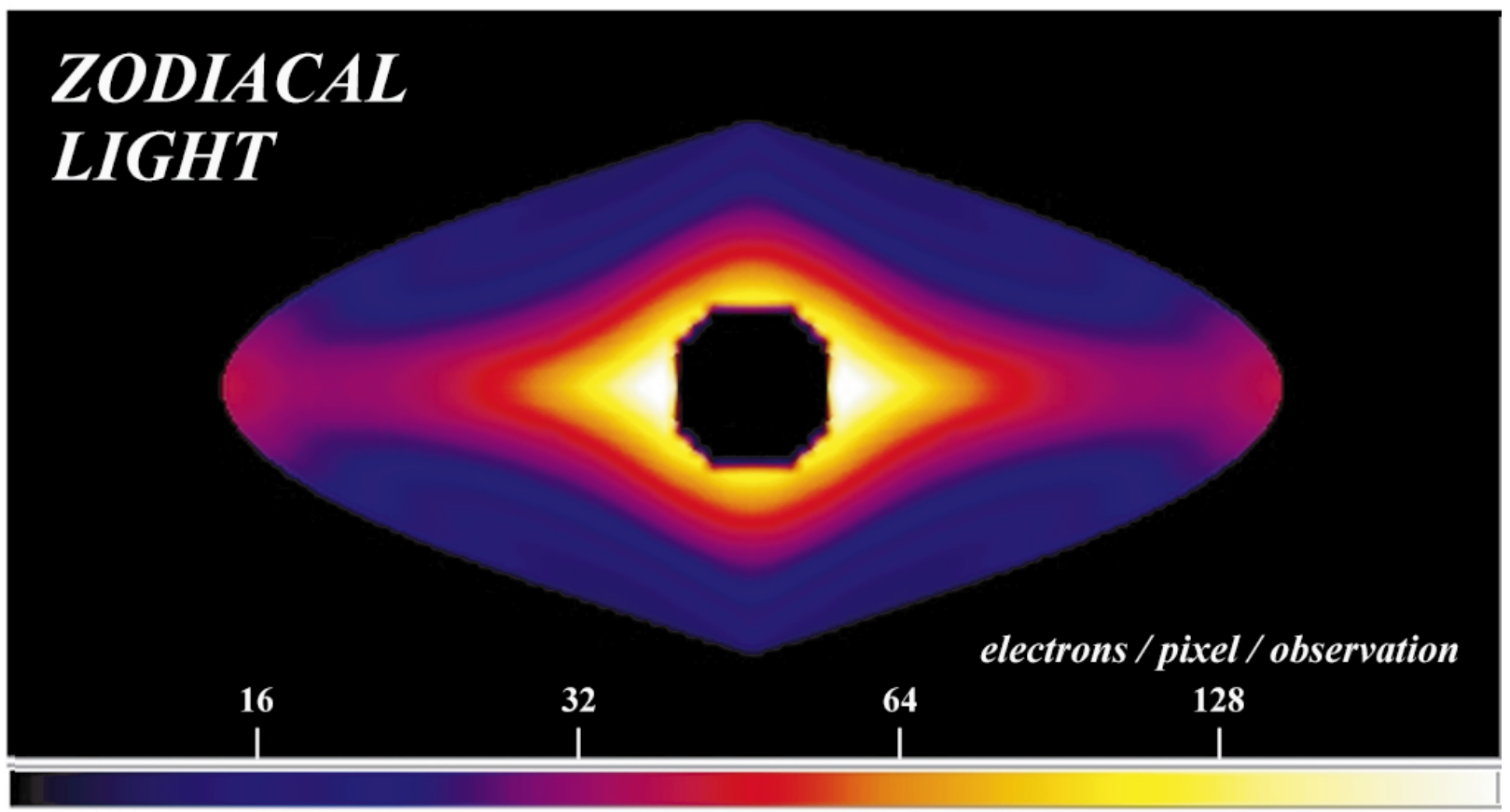

\section{b)}

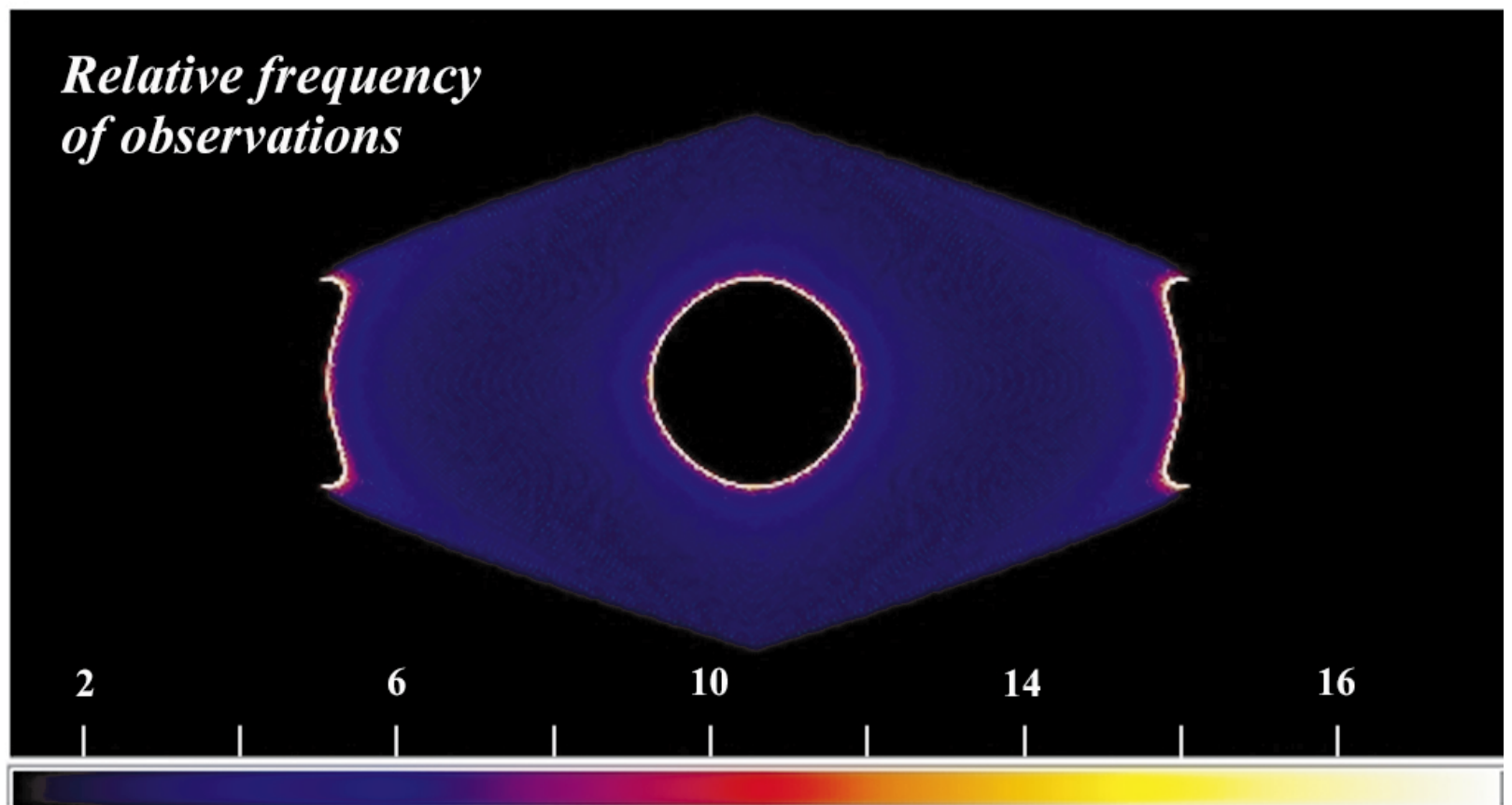

Fig. 3. a) Number of electrons per pixel per transit due to zodiacal light background. The data are shown in helioecliptic coordinates, where the Sun stays at the center of the figure and equator coincides with the ecliptic. b) Relative number of transits in the same coordinates.

presents a standard radial velocity error, $\left[\sum_{\mathrm{i}=1}^{\mathrm{N}}\left(v_{i}(\right.\right.$ true $)-$ $v_{i}($ recovered $\left.\left.)\right)^{2} / N\right]^{0.5}$, as resulting from some $N=400$ tries using a given spectral resolution for a star of a given spectral type and magnitude. The distribution of errors of each point turns out to be Gaussian within statistical errors also for the faintest stars and for all resolutions. The xcsao correlation routine had no appreciable convergence problems even for the faintest stars sampled at the lowest spectral resolution (the convergence was always succesfull in $>95 \%$ of the tries). We used 5 different spectral dispersions, colour coded in Fig. 4. The resolution element had always a size of two pixels. The graphs are in three rows, each for a different type of a star. The left column gives results for a single transit spectrum, while the right one is 


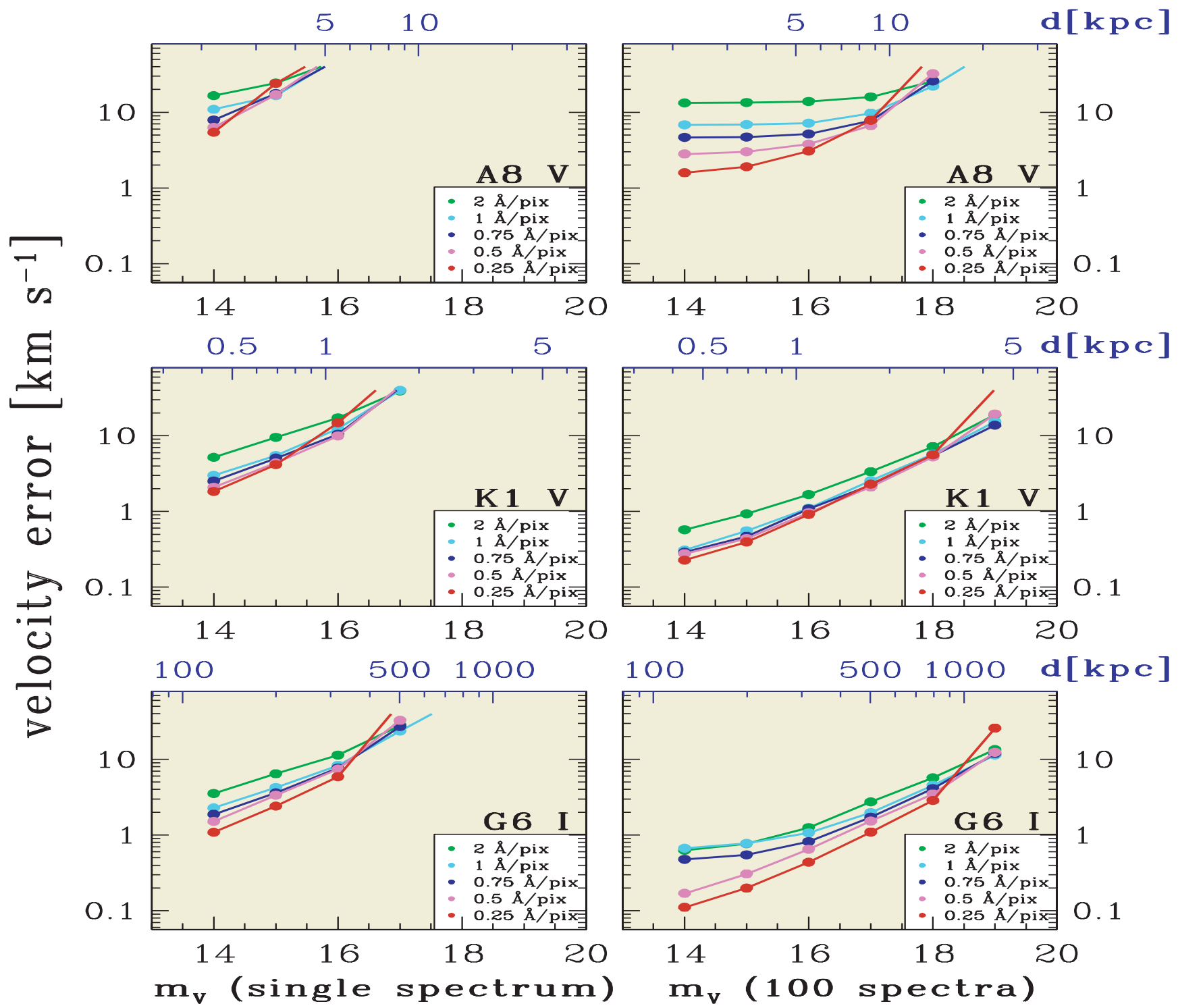

Fig. 4. Average radial velocity determination error as a function of stellar magnitude, spectral type and the used spectral dispersion. Top labels at each graph quote a representative distance if no interstellar absorption is present. Left column is for single transit spectra, while the right one is for a mission-averaged spectrum (100 transits). The assumed background is $74 \mathrm{e}^{-}$per wavelength bin.

for a mission-averaged spectrum. Top labels at each graph quote a representative distance of the star, assuming no interstellar absorption is present.

Simulations presented in Fig. 4 assume the background of $74 \mathrm{e}^{-}$per wavelength bin. This is an average value of zodiacal light background for an object on the ecliptic plane with the spectrum falling on a single pixel row (Fig. 3a) and corresponds to $V=21.53 / \operatorname{arcsec}^{2}$. We also run an identical set of simulations for a lower value of the sky background (the adopted value of $20 \mathrm{e}^{-}$per wavelength bin is an average value for an object $45^{\circ}$ away from the ecliptic).

The results for the three types of stars are remarkably different. Spectrum of a hot A8 V type star $(T=7500 \mathrm{~K}$, $\left.\left[Z / Z_{\odot}\right]=0.0, \log (g)=4.5\right)$ has relatively few lines, so its results are worse than those obtained for cooler stars.
It is dominated by weak and sharp Ca II lines and the Paschen series lines. The relative intensity of the Ca II and Paschen lines rapidly changes with temperature so a mismatch in the spectral type of the star introduces a systematic error in the strength of both sets of lines. The correlation routine tries to compensate for line intensity mismatch by introducing an additional velocity shift that causes a systematic offset in the recovered velocity. This error can be corrected by obtaining spectra with better resolution: better sampling of line profiles means more points carrying radial velocity information, so that the correlation routine can avoid systematic errors in the recovered radial velocity. This is well seen in top-right panel of Fig. 4, where the accuracy of bright star $(V \leq 15)$ measurements depends only on spectral resolution and not on the magnitude. The situation for faint targets $(V \geq 17)$ is 
different: the adopted relatively high background starts to dominate the statistics for the highest resolutions, so dispersions of $0.5 \AA /$ pix or $0.75 \AA /$ pix perform best for faint hot targets. Note that the point of crossing, i.e. the limit where choosing coarser dispersion starts to pay off, depends on the adopted value of the background. For another set of simulations with assumed background value of $20 \mathrm{e}^{-}$(Table 2 ) the crossing occurs only at magnitude $V=18.0$.

Cool dwarfs $\left(\mathrm{K} 1 / 2 \mathrm{~V}: T=5000 \mathrm{~K},\left[Z / Z_{\odot}\right]=0.0\right.$, $\log (g)=4.5)$ perform better. The reason are very strong, core-saturated Ca II lines that dominate the spectrum. These lines enable correlation at fainter magnitudes, so improving the accuracy. And because Ca II lines are very broad and symmetrical the issue of resolution is less important. Actually the radial velocity accuracy is very similar for all dispersions except for the coarsest one $(2 \AA /$ pix $)$ where even the broad Ca II lines get undersampled. The crossing of the $0.25 \AA /$ pix dispersion occurs at $V \sim 15.5$ (spectrum from a single transit) and $V \sim 18$ (mission average). If the background were lower $\left(20 \mathrm{e}^{-}\right.$, Table 2$)$ the errors at the faint end would be $50 \%$ lower and the crossing less pronounced. The results for a mission average spectrum are very similar to those of $\sim 2.5$ mag brighter stars observed during a single transit. If the spectrum had no background and readout noise limits, the gain would be a factor of 100 in flux or a difference of 5 mag.

Cool supergiants (G6 I: $T=4750 \mathrm{~K},\left[Z / Z_{\odot}\right]=0.0$, $\log (g)=1.0)$ will be optimal targets for deriving accurate radial velocities at extreme distances. Their spectra are much richer than those of dwarfs of similar temperature, as the Paschen lines and many lines of metals start to appear. More lines mean more points that can constrain the position of the peak of the correlation function. As a result the radial velocity is derived with remarkable accuracy. The question of resolution is important: better resolution allows for distinction of the $\mathrm{Ca}$ II and Pashen lines, as well as deblending of close metallic lines. The crossing of the $0.25 \AA /$ pix dispersion occurs at $V \sim 16.3$ for single transit spectra, while the highest resolution still performs well even at magnitude $V=18$ for a mission averaged spectrum. The reason is the richness of lines so that co-addition of many well-sampled spectra is still advantageous. On the other hand weak lines in faint stars get lost in the noise, so the question of a chosen spectral resolution is less critical at the faint end than it is for bright stars. In the case of low background $\left(20 \mathrm{e}^{-}\right)$the errors for the faint stars are $50 \%$ lower.

Figure 4 demonstrates that radial velocities with an accuracy of $2 \mathrm{~km} \mathrm{~s}^{-1}$ in A8 dwarfs are obtainable only for the case of the highest spectral resolution $(0.25 \AA /$ pix $)$. These stars can be observed up to the distance of $\sim 5 \mathrm{kpc}$. For cooler dwarfs $(\mathrm{K} 1 / 2 \mathrm{~V})$ the same accuracy is achievable for fainter targets $(V \leq 17)$, corresponding to smaller distances $(\sim 2 \mathrm{kpc})$. The issue of spectral dispersion is less important than for hot dwarfs unless the worst sampling $(2 \AA /$ pix $)$ is chosen. Note that accuracy achievable from single transit spectra is important: most spectroscopic binaries or intrinsically variable (spot) stars will be cool dwarfs. Figure 4 shows that GAIA will be able to measure their radial velocity down to $V=15$ with an error of $\sim 3 \mathrm{~km} \mathrm{~s}^{-1}$. But the superposition of various dispersions can be misleading in this case: spectroscopic binaries or spotty stars require a sufficient spectral resolution for any meaningful measurement (Munari et al. 2001a, 2002). The cool supergiants can be used to measure kinematics at the largest distances, reaching nearby galaxies.

Tables 1 and 2 give detailed results for two levels of the background. Relative uncertainties of the quoted standard velocity errors are estimated at $3 \%$, as can be seen from comparison of values for bright stars in both tables. We added results for classical Cepheids $(T=5500 \mathrm{~K}$, $\left.\left[Z / Z_{\odot}\right]=-0.2, \log (g)=2.0, v \sin i=10 \mathrm{~km} \mathrm{~s}^{-1}\right)$ and horizontal branch stars $\left(T=5000 \mathrm{~K},\left[Z / Z_{\odot}\right]=-0.5\right.$, $\left.\log (g)=3.5, v \sin i=10 \mathrm{~km} \mathrm{~s}^{-1}\right)$ that are important kinematic tracers in the Galaxy (ESA 2000). Results can be readily scaled to any ground based spectrograph with collecting area different from $0.53 \mathrm{~m}^{2}$ as the single transit spectra have an effective exposure time of $1 \mathrm{~min}$ and the mission averaged ones $100 \mathrm{~min}$.

\section{Discussion}

The results presented in Fig. 4 are compatible with initial performance assessment of the GAIA radial velocity spectrometer (Katz 2000). Recently Munari et al. (2001) published an extensive series of cross-correlations using an observed set of Echelle spectra. They limited themselves to stars brighter than those considered here. But bright stars of identical spectral type are hard to find. So they had to use a large spread of spectral types, and the actual mismatch between spectra and templates was larger than will be for the mission. Correspondingly they derived somewhat larger radial velocity errors (within a factor of 2). But they came to the same basic conclusion: a high-enough spectral dispersion is a critical factor determining radial velocity accuracy for bright stars.

Here we extend this work to fainter magnitudes. We explore a range of spectral types and include simulations of very faint stars having a signal to noise ratio for a single transit $S / N<1$. At such extreme conditions a spectrum with the dispersion of $0.25 \AA /$ pix is limited by the sky background. And because this background is mostly zodiacal light a somewhat lower dispersion $(0.5 \AA /$ pix $)$ performs better for the faintest $(V \geq 18)$ dwarfs. The resolution of $0.75 \AA /$ pix is also acceptable for the faintest stars.

There are other reasons to collect high dispersion spectra. Figure 1 shows that values of all spectral parameters can be accounted for by spectroscopy, but note that a high spectral resolution is needed to make secure distinctions. All tracings are plot for $R=20000$; halving the resolution already mixes up gravity and rotation or metallicity and $\alpha$-element enrichment. Additional set of simulations shows that $\alpha$-element enrichment of +0.4 can be detected down to $V=14$, and abundancies of individual elements can 
Table 3. Estimate of expected crowding of the spectra. See text.

\begin{tabular}{lrrrr}
\hline \hline & \multicolumn{2}{c}{$V<20$} & \multicolumn{2}{c}{$V<17$} \\
& $|b|<20^{\circ}$ & $|b|>20^{\circ}$ & $|b|<20^{\circ}$ & $|b|>20^{\circ}$ \\
\hline$n$ [stars/ deg. ${ }^{2}$ ] & 61000 & 5600 & 6100 & 1200 \\
aver. separation & $15^{\prime \prime}$ & $48^{\prime \prime}$ & $46^{\prime \prime}$ & $104^{\prime \prime}$ \\
free length $(l)$ & $212^{\prime \prime}$ & $2310^{\prime \prime}$ & $2120^{\prime \prime}$ & $10800^{\prime \prime}$ \\
\hline
\end{tabular}

be measured for bright stars. Abundance of a particularly difficult one, Manganese (with the only useful line in the GAIA spectral range at $\lambda=8740.9 \AA$ with $E W \approx 0.02 \AA$ ) can be determined within a factor of 2 for $V<8$. Other elements, i.e. N, Si, Fe, Mg and Ti cause much stronger lines and so can be studied down to $V \approx 13$. Stellar rotation is easy to determine for fast rotators (Munari 1999), but cool dwarfs commonly observed by GAIA rotate slowly. Stellar rotation with an error of $10 \mathrm{~km} \mathrm{~s}^{-1}$ of a $T=5000 \mathrm{~K}$ dwarf can be measured for $V<9$. All results quoted here are for the dispersion of $0.25 \AA /$ pix and depend critically on the spectral resolution.

GAIA will observe many spectroscopic binaries. Munari et al. (2001a) showed that good quality orbital solutions, including masses, can be derived. But also this result critically depends on the resolution of the spectrum. Single Angstrom corresponds to $35 \mathrm{~km} \mathrm{~s}^{-1}$ at the wavelengths of the GAIA spectrograph. Velocity amplitudes of most spectroscopic binaries are not much larger than this, so it is clear that a dispersion of 0.25 or $0.5 \AA /$ pix is needed for a proper deblending of lines from both stars.

The Galaxy model (Torra et al. 1999) using Hakkila et al. (1997) extinction law predicts an average surface density $n$ of stars (Table 3 ). This can be translated into an average stellar separation $\left(n^{-0.5}\right)$. Two spectra are blended if their spectral tracings are separated by less than the spatial extent $s$ of a CCD pixel, i.e. 1 arcsec. This happens if the length of the spectral tracing is larger than the free length $l=(n s)^{-1}$. The length of the tracing depends on the dispersion and equals 1000 arcsec for $0.25 \AA /$ pix, 500 arcsec for $0.5 \AA /$ pix and 333 arcsec for $0.75 \AA /$ pix. So Table 3 demonstrates that spectral superposition will be common close to the galactic plane, but not away from it.

Light of contaminating stars will be spread over many pixels, so the zodiacal light will usually remain the dominant background signal. Stellar background features stellar lines and this could influence the radial velocity accuracy. Still, extremely accurate astrometry and photometry of contaminating stars will supply enough information to handle the problem. In the worst cases the situation will be similar to disentangling the two spectra in a spectroscopic binary. But with an extra benefit that spectral overlap varies from transit to transit. So, even if two spectra are badly superimposed, the same two stars will be recorded separately during the next transit.

\section{Conclusions}

We studied performance of a slitless spectrograph observing in a $250 \AA$ window centered at $8615 \AA$ that will be used aboard GAIA. The same spectral range is accessible also from the ground, but cannot be much expanded, due to bracketing telluric absorptions. So the results are relevant also for planning of ground based instruments.

The dominant parameter that can still be changed is the spectral resolution. We used realistic estimates of zodiacal light background and spectrograph parameters to derive expected accuracy of the radial velocity determination. We showed that errors below $2 \mathrm{~km} \mathrm{~s}^{-1}$ are achievable for bright enough stars if a high dispersion is chosen. This result could be still improved with correlation methods that use software spectral masks (Baranne et al. 1979; Queloz 1995).

The results show that it is worth to observe very faint targets, down to magnitude 19 even though the expected radial velocity errors can be well above $10 \mathrm{~km} \mathrm{~s}^{-1}$. Such stars are numerous. One may expect a ratio of $\sim 10: 1$ in the recorded number of targets between $V=17$ and 19 compared to stars between $V=14$ and 16. For such stars the best results are obtained with $0.5 \AA /$ pix or $0.75 \AA /$ pix dispersion. But for brighter stars a high dispersion $(0.25 \AA /$ pix $)$ is always a preferred choice and is critical in order to extract relevant information for nontrivial cases, as spectroscopic binaries, variable stars and peculiar stars.

It remains to be determined if telemetry constraints permit to use the dispersions of 0.25 or $0.5 \AA /$ pix. Still it is clear that such a choice has a strong support considering the astrophysical information retrievable from the data.

Acknowledgements. The author wishes to thank U. Munari for initial motivation and many fruitful discussions and to F. Castelli for an introduction to the art of Kurucz models. The referee, D. Katz, is acknowledged for useful comments which improved the quality of the paper. This work was supported by a grant from the Slovenian Ministry of Education, Science and Sport.

\section{References}

Baranne, A., Mayor, M., \& Poncet, J. L. 1979, Vistas in Astronomy, 23, 279

Castelli, F., \& Munari, U. 2001, A\&A, 366, 1003

de Zeeuw, P. T., Gilmore, G., Perryman, M. A. C., et al. 2000, GAIA - Composition, Formation and Evolution of the Galaxy, ESA-SCI(2000)4, [ESA 2000]

Frey, A., Hoffmann, W., Lemke, D., \& Thum, C. 1974, A\&A, 36,447

Garcia, B. 1989, CDS Catalog, 3149

Gorkavyi, N., Ozernoy, L., Mather, J., \& Taidakova, T. 2000, in Next Generation Space Telescope Science and Technology, ed. E. Smith, \& K. Long, ASP Conf. Ser., 207, 462

Hakkila, J., Myers, J. M., Stidham, B. J., \& Hartmann, D. H. 1997, AJ, 114, 2043

Katz, D. 2000, Ph.D. Thesis, Univ. Paris 7

Kelsall, T., Weiland, J. L., Franz, B. A., et al. 1998, ApJ, 508, 44 
James, F., Mukai, T., Watanabe, T., Ishiguro, M., \& Nakamura, R. 1997, MNRAS, 288, 1022

Levasseur-Regourd, A. C., \& Dumont, R. 1980, A\&A, 84, 277

Kurtz, M. J., Mink, D. J., Wyatt, W. F., et al. 1992, in Astronomical data analysis software and systems, ed. D. M. Worrall, C. Biemesderfer, \& J. Barneseds, ASP Conf. Ser., 25,432

Matsumoto, T., Kawada, M., Murakami, H., et al. 1996, PASJ, 48, L47

Munari, U. 1999, in Proceedings of the ESA Leiden Workshop on GAIA, 23-27 Nov. 1998, Baltic Astronomy, 8, 73

Munari, U., \& Tomasella, L. 1999, A\&AS, 137, 521

Munari, U., \& Castelli, F. 2000, A\&AS, 141, 141

Munari, U. 2001, in GAIA, an European Space Project, Proc. of Les Houches 2001 summer sch., EDP Sciences, in press [astro-ph/0109057]
Munari, U., Agnolin, P., \& Tomasella, L. 2001, Baltic Astronomy, 10, 613

Munari, U., Tomov, T., Zwitter, T., et al. 2001a, A\&A, 378, 477

Munari, U., Tomasella, L., Marrese, P. M., et al. 2002, A\&A, submitted

Perryman, M. A. C., de Boer, K. S., Gilmore, G., et al. 2001, A\&A, 369, 339

Queloz, D. 1995, in New developments in array technology and applications, IAU Symp., 167 (Kluwer), 221

Tonry, J., \& Davis, M. 1979, AJ, 84, 1511

Torra, J., Chen, B., Figuera, F., Jordi, C., \& Luri, X. 1999, in Proceedings of the ESA Leiden Workshop on GAIA, 23-27 Nov. 1998, Baltic Astronomy, 8, 171

Zwitter, T., Munari, U., \& Castelli, F. 2002, A\&A, submitted 